Document downloaded from:

http://hdl.handle.net/10251/171219

This paper must be cited as:

Sanz Diaz, R.; García Gil, PJ.; Diez, J.; Bondía Company, J. (2021). Artificial Pancreas System With Unannounced Meals Based on a Disturbance Observer and Feedforward Compensation. IEEE Transactions on Control Systems Technology. 29(1):454-460. https://doi.org/10.1109/TCST.2020.2975147

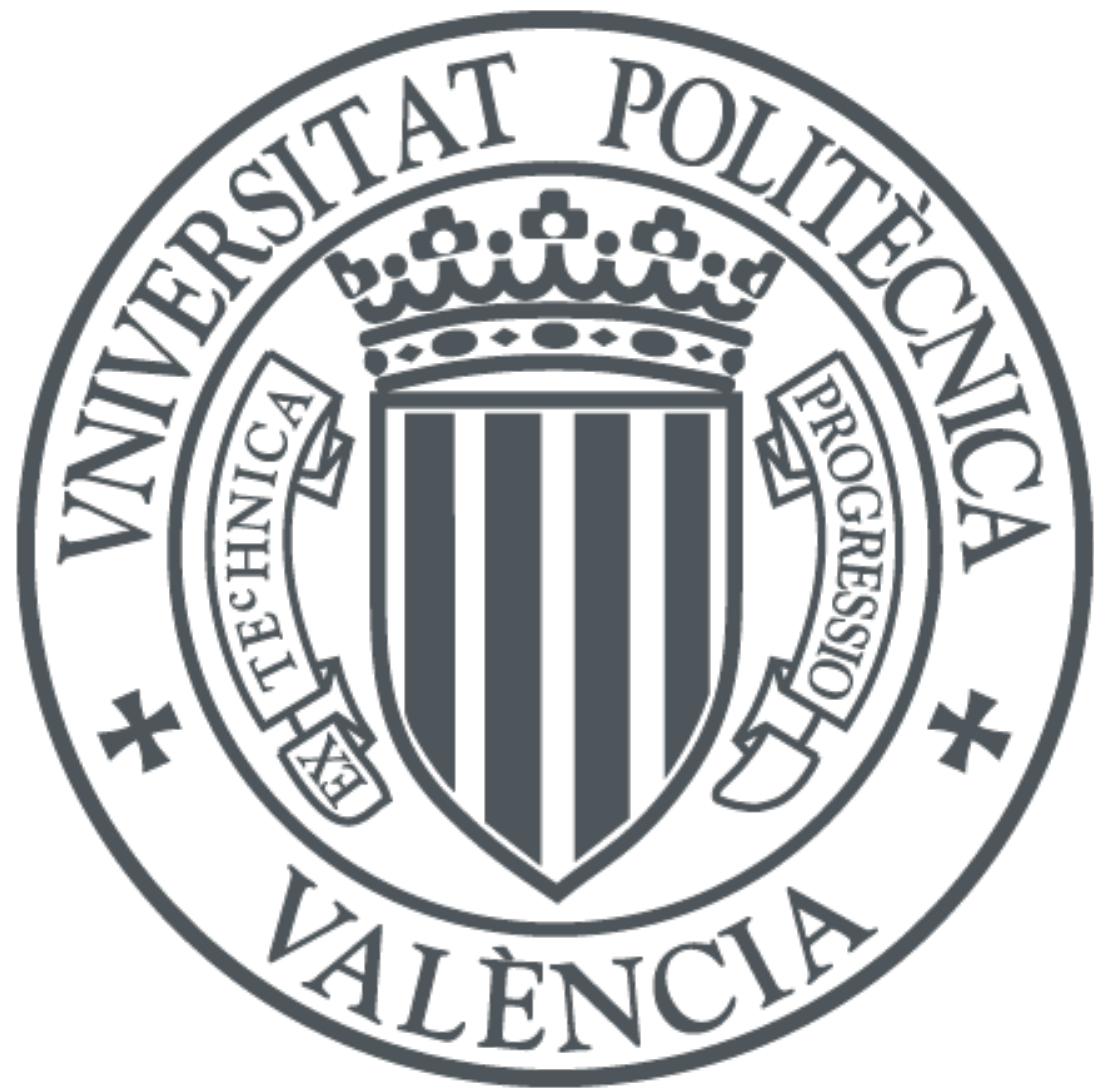

The final publication is available at

https://doi.org/10.1109/TCST.2020.2975147

Copyright Institute of Electrical and Electronics Engineers

Additional Information

(C) 2021 IEEE. Personal use of this material is permitted. Permissíon from IEEE must be obtained for all other uses, in any current or future media, including reprinting/republishing this material for advertisíng or promotional purposes, creating new collective works, for resale or redistribution to servers or lists, or reuse of any copyrighted component of this work in other works. 


\title{
Artificial Pancreas System with Unannounced Meals based on a Disturbance Observer and Feedforward Compensation
}

\author{
Ricardo Sanz, Pedro García, José-Luis Díez and Jorge Bondia
}

\begin{abstract}
This paper is focused on closed-loop control of postprandial glucose levels of patients with type 1 diabetes mellitus after unannounced meals, still a major challenge towards a fully autonomous artificial pancreas. The main limitations are the delays introduced by the subcutaneous insulin pharmacokinetics and the glucose sensor, which typically lead to insulin over-delivery. Current solutions reported in the literature typically resort to meal announcement, which requires the patient intervention. In this paper, a disturbance observer is used to estimate the effect of unannounced meals and the insulin pharmacokinetics is taken into account by means of a feedforward compensator. The proposed strategy is validated in silico with the UVa/Padova metabolic simulator. It is demonstrated how the disturbance observer successfully estimates and counteracts not only the effect of meals but also sudden drops in the glucose levels that may lead to hypoglycemia. For unannounced meals, results show a median time-in-range of $80 \%$ in a 30-day scenario with high carbohydrate content and large intra-subject variability. Optionally, users may decide to announce meals. In this case, considering severe bolus mismatch due to carbohydrate counting errors, the median time-in-range is increased up to $88 \%$. In every case, hypoglycemia is avoided.
\end{abstract}

Index Terms-Artificial pancreas, disturbance observer, feedforward compensation, type 1 diabetes

\section{INTRODUCTION}

$\mathbf{T}$ YPE 1 diabetes Mellitus (T1DM) is an auto-immune disorder that destroys the pancreatic $\beta$ cells, which results in the incapability of secreting insulin, a hormone that plays a crucial role in glucose homeostasis as it is responsible of lowering plasma glucose concentration. Therefore, people with T1DM generally fail to maintain appropriate glucose levels and they tend to suffer chronic hyperglycemia, which leads to

This work was partially supported by Ministerio de Economía y Competitividad (DPI2016-78831-C2-1-R) and the European Union through FEDER funds. (Corresponding Author: J. Bondia)

R. Sanz is with the Instituto Universitario de Automática e Informática Industrial, Universitat Politècnica de València, 46022, València, Spain (e-mail: risanda@upv.es)

P. García is with the Instituto Universitario de Automática e Informática Industrial, Universitat Politècnica de València, 46022, València, Spain (e-mail: pggil@isa.upv.es)

J. L. Díez is with the Instituto Universitario de Automática e Informática Industrial, Universitat Politècnica de València, 46022, València, Spain, and also with the Centro de Investigación Biomédica en Red de Diabetes y Enfermedades Metabólicas Asociadas (CIBERDEM), 28029, Madrid, Spain (e-mail: jldiez@isa.upv.es)

J. Bondia is with the Instituto Universitario de Automática e Informática Industrial, Universitat Politècnica de València, 46022, València, Spain, and also with the Centro de Investigación Biomédica en Red de Diabetes y Enfermedades Metabólicas Asociadas (CIBERDEM), 28029, Madrid, Spain (e-mail: jbondia@isa.upv.es) severe health problems in the long term, e.g., cardiovascular diseases, nephropathy, retinopathy and neuropathy [1]. This is the reason why exogenous insulin delivery is needed. Openloop (manual) control by individuals with T1DM is highly demanding as it requires carbohydrate counting and a great number of insulin dosing decisions per day. It is not surprising then that closed-loop (automatized) insulin delivery has been an active research field since the 1970s [2], [3], [4]. The device that performs such task is widely known today as an artificial pancreas (AP).

In an $\mathrm{AP}$, the insulin dose is computed by a control algorithm based on glucose measurements coming from a continuous glucose monitoring (CGM) device and automatically delivered through an insulin pump [5]. There is a substantial delay between the subcutaneous insulin delivery, its appearance in the blood stream and, ultimately, its effect in lowering blood glucose levels. Such long delay typically causes a feedback controller to over-deliver insulin, resulting in hypoglycemia, which is the main risk of closed-loop glucose control [6]. Nevertheless, the improvement of an AP in glucose regulation compared to open-loop therapy is widely acknowledged [7], [8], [9], [10]. A wide variety of control strategies have been applied to tackle this problem. Controllers based on model predictive control (MPC) [11], [12], [13], [14], proportional-integral-derivative (PID) control [15], [16], fuzzy logic [17], [18] or linear parameter varying (LPV) models [19], [20] have been tested in human trials. However, mitigation by the AP of hyperglycemia due to large meals is still challenging because of the limitations discussed above. Quite often, current APs deal with this problem by delivering an insulin bolus at meal time. However, this requires the patient intervention, who has to announce the meal and provide an estimate of its carbohydrate $(\mathrm{CHO})$ content. Misestimation of $\mathrm{CHO}$ content and/or skipping meal announcements is indeed a risk and thus the controller should be able to handle these situations. In order to face unannounced meals, heuristic meal detection and carbohydrate counting algorithms based on CGM readings have been investigated, which are often used to deliver meal boluses accordingly [21], [22], [23], [24], [25]. In [19], a meal detection algorithm is used to trigger a switching controller. Within the framework of MPC, a switching cost-function based on the blood glucose rate of change has been employed in [26].

In the context of control theory, maintaining blood glucose near the target level can be seen as a standard regulation problem, in which disturbance rejection is the central issue. 
When disturbances are known, their effect can be mitigated by means of feedforward compensators [27]. However, quite often, disturbances cannot be directly measured (for example, meals in the application here considered). Disturbance observers (DOBs) emerged as a powerful tool to estimate unknown disturbances from other measurable input/output signals. Then, the estimated disturbance can be used to take appropriate actions, leading to the so-called DOB-based control. The reader is referred to [28] for an overview of available methods. DOB-based controllers have demonstrated simpler tuning and improved performance over equivalent PID-based strategies even in high-demanding real-time applications [29]. Furthermore, DOBs to estimate the rate of glucose appearance have been already designed and successfully validated through simulations in [30], [31].

In this paper, a DOB-based control strategy is proposed to deal with unannounced meals. The observer estimates any unexpected variation in the glucose level by using information of the CGM, the insulin infusion rate and a control-oriented nonlinear model of the patient adapted from [12], [32], [33]. The estimation provided by the observer is then combined with a feedforward strategy to compensate for the delay of the subcutaneous insulin pharmacokinetics. On the other hand, in order to handle the constraint of positive control action and to avoid insulin over-delivery, the insulin feedback strategy reported in [6] is employed.

\section{Methods}

\section{A. Control-oriented patient model}

A subject-dependent model is employed for control purposes, adapted from [12], [32]. For each subject $j$, the following switched nonlinear personalized model is considered

$$
\begin{aligned}
\dot{G}(t) & =-\left(S_{G}(G)+r(G) X(t)\right) G(t)+S_{G}(G) G_{b}+R_{a}(t), \\
\dot{X}(t) & =-p_{2} X(t)+p_{2} S_{I} c_{j} I(t), \\
\dot{I}(t) & =-k_{I} I(t)+\left(1 /\left(t_{\max _{I}} V_{I}\right)\right) S_{2}(t), \\
\dot{S}_{2}(t) & =-\left(1 / t_{\text {max }_{I}}\right) S_{2}(t)+\left(1 / t_{\max _{I}}\right) S_{1}(t), \\
\dot{S}_{1}(t) & =-\left(1 / t_{\text {max }_{I}}\right) S_{1}(t)+\bar{u}_{j}(t),
\end{aligned}
$$

where $G(\mathrm{mg} / \mathrm{dL})$ is the plasma glucose concentration, $R_{a}$ $(\mathrm{mg} / \mathrm{dL} / \mathrm{min})$ is the rate of glucose appearance, $X(1 / \mathrm{min})$ is the remote insulin action, $I(\mu \mathrm{U} / \mathrm{mL})$ is the deviation in plasma insulin concentration, $S_{2}, S_{1}(\mu \mathrm{U} / \mathrm{kg})$ are the deviations in insulin amount at each compartment of the absorption model and $\bar{u}_{j}(\mu \mathrm{U} / \mathrm{kg} / \mathrm{min})$ is the incremental infusion rate with respect to the basal insulin infusion $u_{b_{j}}$, needed to maintain the fasting glucose value $G_{b}$. The functions $r, S_{G}: \mathbb{R}^{+} \rightarrow \mathbb{R}^{+}$are defined by $r(G)=\{\bar{r}$ if $G \geq G_{b} ; \underline{r}$ if $\left.G<G_{b}\right\}$ and $S_{G}(G)=\left\{\bar{S}_{G}\right.$ if $G \geq G_{b} ; \underline{S}_{G}$ if $G<G_{b}$, which are intended to account for the increase of the insulin action when glucose decreases under a given threshold towards hypoglycemia (this is a simplification of the risk function reported in [33]). The individualized parameter $c_{j}$ is used to adapt the model gain as

$$
c_{j}=\max \left\{0.7, \min \left\{\mathrm{CR}_{j} / \overline{\mathrm{CR}}, \mathrm{CF}_{j} / \overline{\mathrm{CF}}\right\}\right\},
$$

where $\mathrm{CR}_{j}, \mathrm{CF}_{j}$ are, respectively, the individual insulin-tocarbohydrate ratio, given by the simulator, and the correction factor, computed upon the total daily insulin (TDI) as $\mathrm{CF}_{j}=1800 / \mathrm{TDI}$ (this is referred to as the 1800-rule, often used in conventinal diabetes therapy [34]); and $\overline{\mathrm{CR}}=$ $15.9 \mathrm{~g} / \mathrm{U}, \overline{\mathrm{CF}}=40.8 \mathrm{mg} / \mathrm{dL} / \mathrm{U}$ are the mean population values based on the ten adult virtual subjects of the distribution version of the UVa/Padova simulator. The factor $c_{j}$ is lower bounded by 0.7 for safety to avoid too aggressive controllers. It should be stressed that the use of compact models for controller design purposes is well celebrated in the field [35], [36]. More specifically, gain-personalized models have been previously used in the literature as a useful tool to account for the variability of total daily insulin requirement among patients [37], [14], [38]. Moreover, $\mathrm{CF}_{j}$ and $\mathrm{CR}_{j}$ are clinical parameters that are known for every patient and thus the proposed tuning rule would be feasible in a clinical trial.

\section{B. Model identification}

A data set for identification was generated using the UVa/Padova simulator. This data set is based on an openloop therapy and it includes meals of different size, bolusing and changes in the basal rate adjustment, including sinusoidal variations. Each of the ten adult virtual patients was identified using the genetic algorithm provided by Matlab ${ }^{\circledR}$ and population values were obtained by averaging them. The value $\bar{r}=1$ was set manually to avoid identification problems because, otherwise, the effect of the insulin sensitivity $S_{I}$ and the free parameters $\underline{r}, \bar{r}$ would be indistinguishable. The following mean values were obtained: $t_{\max _{I}}=48.8 \mathrm{~min}, k_{I}=$ $0.58 \mathrm{~min}^{-1}, V_{I}=28.4 \mathrm{~mL} / \mathrm{Kg}, S_{I}=6 \cdot 10^{-4} \mathrm{~mL} / \mu \mathrm{U} / \mathrm{min}$, $p_{2}=0.027 \mathrm{~min}^{-1}, \bar{S}_{G}=0.017 \mathrm{~min}^{-1}, \underline{S}_{G}=0.007 \mathrm{~min}^{-1}$ and $\underline{r}=1.41$. It should be remarked that $G_{b}$ is a control parameter defining the operating coditions in the absence of disturbances, which was set to $G_{b}=100 \mathrm{mg} / \mathrm{dL}$, and the corresponding basal insulin infusions for each patient, $u_{b_{j}}$, are obtained from the equilibrium equations of the simulator model [33].

\section{Control strategy}

The system (1) can be seen as the interconnection between a nonlinear subsystem, that is, the glucose metabolism, and a linear subsystem, namely, the insulin pharmacokinetics. According to this decomposition and defining

$$
x(t)=\left[X(t), I(t), S_{2}(t), S_{1}(t)\right]^{T},
$$

the model (1) can be expressed as

$$
\begin{aligned}
\dot{G}(t) & =f\left(G(t), x_{1}(t), R_{a}(t)\right), \\
\dot{x}(t) & =A x(t)+B \bar{u}_{j}(t), \\
y(t) & =x_{1}(t)=X(t),
\end{aligned}
$$

where the function $f$ and the matrices $A, B$ are easily obtained from (1). For the sake of clarity, let us assume for the moment that both the state $x$, and the rate of glucose appearance $R_{a}(t)$, are known. 
The proposed controller has a cascade structure. First, by looking at the nonlinear subsystem, the following exponentially stable target system ${ }^{1}$ given by $\dot{G}(t)=-S_{G}(G) G(t)+$ $S_{G}(G) G_{r e f}(t)$, is proposed, which can be achieved by choosing a target trajectory $x_{1}^{*}(t)$ as follows

$$
x_{1}^{*}(t)=\frac{1}{G(t) r(G)}\left(R_{a}(t)+S_{G}(G)\left(G_{b}-G_{r e f}(t)\right)\right),
$$

being $G_{r e f}(t)$ a piecewise constant function representing the desired glucose concentration. Second, a tracking controller is designed so that the error $e(t)=x_{1}(t)-x_{1}^{*}(t)$ approaches to zero exponentially. To that end, let us consider the transformation $z(t)=T x(t)$ so that the system in $z$-coordinates, given by $\dot{z}(t)=A_{z} z(t)+B_{z} \bar{u}_{j}(t)$, is in controllable canonical form, i.e.,

$$
A_{z}=\left[\begin{array}{cccc}
0 & 1 & 0 & 0 \\
0 & 0 & 1 & 0 \\
0 & 0 & 0 & 1 \\
-a_{1} & -a_{2} & -a_{3} & -a_{4}
\end{array}\right], \quad B_{z}=\left[\begin{array}{l}
0 \\
0 \\
0 \\
b
\end{array}\right]
$$

and $y(t)=z_{1}(t)=x_{1}(t)=X(t)$. It is well known that the transformation matrix is given by $T=\mathcal{C}\left(A_{z}, B_{z}\right) \mathcal{C}(A, B)^{-1}$, where $\mathcal{C}$ denotes the controllability matrix [40]. Once in controllable canonical form, it is easy to design the following tracking controller

$$
\begin{aligned}
\bar{u}_{j}(t)= & \frac{1}{b}\left(a_{4} z_{4}(t)+a_{3} z_{3}(t)+a_{2} z_{2}(t)+a_{1} z_{1}\right) \\
& +\frac{1}{b}\left(x_{1}^{*^{(4)}}(t)-\sum_{j=1}^{4} k_{j}\left(z_{j}(t)-x_{1}^{*^{(j-1)}}(t)\right)\right),
\end{aligned}
$$

that leads to the exponentially stable tracking error system

$$
e^{(4)}(t)+k_{4} e^{(3)}(t)+k_{3} e^{(2)}(t)+k_{2} e^{(1)}(t)+k_{1} e(t)=0,
$$

for any set of gains $k_{j}>0$. The overall stability of the $(\tilde{G}, e)$ system, being $\tilde{G}(t)=G(t)-G_{r e f}(t)$, has a rather common cascade structure where the exponentially stable $e$-subsystem drives the $\tilde{G}$-subsystem. The latter can be cast into a switched system with vanishing perturbations whose stability is proved by means of a common Lyapunov function [41], [42].

\section{Extended state observer}

The controller strategy described above relies on the knowledge of the whole state $x$ and the disturbance $R_{a}$. Since these variables are not measurable in practice, an extended state observer is adopted to estimate them [28], [30]. Using the notation introduced in (3), the proposed observer is given by

$$
\left[\begin{array}{c}
\dot{\hat{G}}(t) \\
\dot{\hat{x}}(t) \\
\dot{\hat{R}}_{a}(t)
\end{array}\right]=\left[\begin{array}{c}
f\left(\hat{G}, \hat{x}_{1}, \hat{R}_{a}\right) \\
A \hat{x}(t)+B \bar{u}_{j}(t) \\
0
\end{array}\right]+L(G)(y(t)-\hat{G}(t))
$$

where $\hat{G}, \hat{x}, \hat{R}_{a}$ are the observer estimates and $L: \mathbb{R} \rightarrow \mathbb{R}^{6}$ is the matrix gain to be designed. The proposed observer (8) is composed of a nonlinear model with a switched linear gain,

\footnotetext{
${ }^{1}$ This is a scalar switched system with two exponentially stable modes whose exponential stability under arbitrary switching is easily proved [39].
}

$L(G)=\left\{\bar{L}\right.$ if $G \geq G_{b} ; \underline{L}$ if $\left.G<G_{b}\right\}$. Each of the gains is designed by pole placement using the corresponding linearized model around $G_{b} \pm \epsilon$ with $\epsilon \rightarrow 0$. The closed-loop poles of the observer are selected according to [30], in order to avoid overshoot in the estimation.

\section{E. Tracking differentiator}

One can see from (6) that the time-derivatives of the target signal $x_{1}^{*}(t)$ are also needed. Instead of computing those numerically, they are rather obtained by means of a simple linear tracking differentiator [43], given by

$$
\dot{\xi}(t)=\left[\begin{array}{cccc}
0 & 1 & 0 & 0 \\
0 & 0 & 1 & 0 \\
0 & 0 & 0 & 1 \\
-\omega^{4} & -4 \omega^{3} & -6 \omega^{2} & -4 \omega
\end{array}\right] \xi(t)+\left[\begin{array}{c}
0 \\
0 \\
0 \\
\omega^{4}
\end{array}\right] x_{1}^{*}(t) \text {. }
$$

It is readily verified that $\lim _{t \rightarrow \infty}\left(\xi_{j}(t)-x_{1}^{*^{(j)}}(t)\right)=0$ with $j \in\{1,2,3,4\}$ for any $\omega>0$, which determines the bandwidth and hence the speed of convergence.

\section{F. Insulin limitation}

The proposed control strategy does not take into account the control input constraint, that is, $0 \leq u(t) \leq u_{\max }$, where $u$ is the absolute insulin infusion rate. Therefore, an aggressive tuning of the controller will result in insulin overdose, which is a known issue in the field of diabetes control [44]. One of the solutions to mitigate this problem is the insulin feedback technique, which consists on the inhibition of the insulin infusion by high values of plasma insulin concentration [6], [45]. It was pointed out in [45] and validated in clinical trials in [46], that the insulin located at the subcutaneous compartments (yet to appear in plasma) could be also included as an inhibition. Following these ideas, the absolute insulin rate delivered by the proposed controller is computed by

$$
\begin{aligned}
u_{j}(t)= & \bar{u}_{j}(t)+u_{b_{j}}-\gamma_{3} \max \left(0, I(t)-\hat{I}_{s s}\right) \\
& -\gamma_{2} \max \left(0, \hat{S}_{2}(t)-S_{2, s s}\right) \\
& -\gamma_{1} \max \left(0, \hat{S}_{1}(t)-S_{1, s s}\right),
\end{aligned}
$$

where $\gamma_{1}, \gamma_{2}, \gamma_{3}>0$ are tuning parameters and $I_{s s}=$ $x_{1, s s} /\left(c_{j} S_{I}\right), S_{2, s s}=t_{\max _{I}} k_{I} V_{I} I_{s s}, S_{1, s s}=S_{2, s s}$ with $x_{1, s s}=S_{G}\left(G_{r e f}\right)\left(G_{b}-G_{r e f}\right) / G_{r e f} / r\left(G_{r e f}\right)$, are the steadystate values corresponding to $G_{r e f}$, which are computed from (1) and (4) assuming $R_{a}(t)=0$.

On the other hand, an upper limit on the maximum insulin infusion rate is imposed in order to avoid insulin over-delivery due to very large meals. In practice, this limit is imposed by the pump capability to deliver insulin and, in any case, it serves as a safety mechanism. A patient-dependent saturation of the control action is proposed so that $u(t) \in\left[0,\left(c_{j} / 0.7\right) u_{\max }\right]$. Recall that $c_{j}$ is the non-dimensional factor used to personalize insulin sensitivity in the patient model (1). The individualized bound allows the patient with lowest $c_{j}$ to exploit the maximum insulin infusion rate $u_{\max }$, and constraints the other patients proportionally. For this application, the value $u_{\max }=16 \mathrm{U} / \mathrm{h}$ is chosen, which corresponds to maximum insulin infusion rate of the Dana Diabecare $\mathrm{R}^{\circledR}$ insulin pump (Sooil, Seoul, Korea). 
TABLE I

MEAL TIMES AND CARBOHYDRATE CONTENT FOR SCENARIOS 1 AND 2

\begin{tabular}{|c|c|c|c|c|c|c|c|c|c|}
\hline & \multicolumn{3}{|c|}{ Day 1} & \multicolumn{3}{|c|}{ Day 2} & \multicolumn{3}{|c|}{ Day 3} \\
\hline Scenario 1 & $7 \mathrm{~h}(50 \mathrm{~g})$ & $14 \mathrm{~h}(60 \mathrm{~g})$ & $20 \mathrm{~h}(50 \mathrm{~g})$ & $6 \mathrm{~h}(50 \mathrm{~g})$ & $13 \mathrm{~h}(70 \mathrm{~g})$ & $19 \mathrm{~h}(50 \mathrm{~g})$ & $7 \mathrm{~h}(50 \mathrm{~g})$ & $13 \mathrm{~h}(65 \mathrm{~g})$ & $21 \mathrm{~h}(55 \mathrm{~g})$ \\
\hline Scenario 2 & $7 \mathrm{~h}(50 \mathrm{~g})$ & $14 \mathrm{~h}(80 \mathrm{~g})$ & $20 \mathrm{~h}(40 \mathrm{~g})$ & $6 \mathrm{~h}(50 \mathrm{~g})$ & $19 \mathrm{~h}(40 \mathrm{~g})$ & $21 \mathrm{~h}(70 \mathrm{~g})$ & $7 \mathrm{~h}(50 \mathrm{~g})$ & $13 \mathrm{~h}(100 \mathrm{~g})$ & $21 \mathrm{~h}(40 \mathrm{~g})$ \\
\hline
\end{tabular}
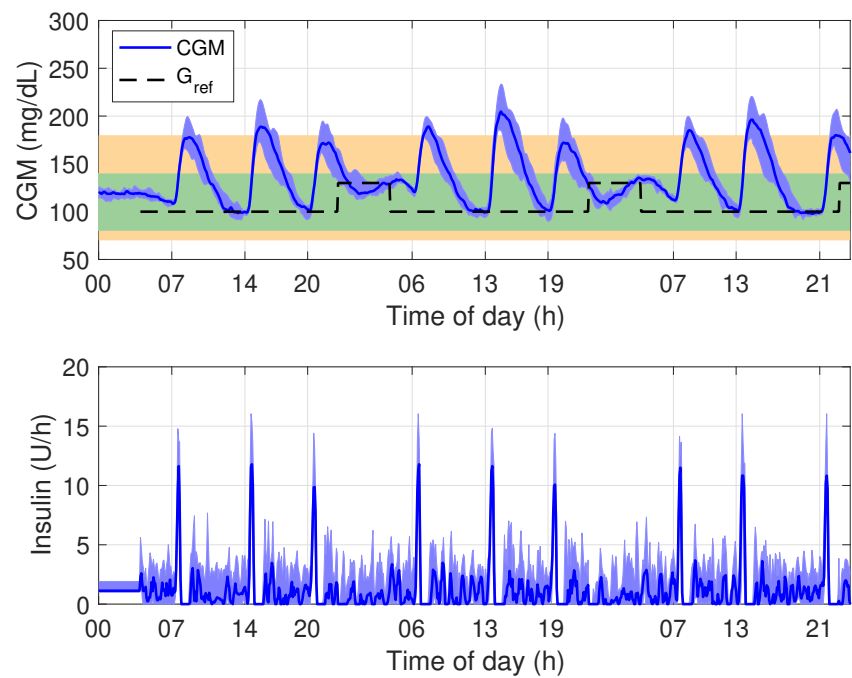

Fig. 1. Closed-loop responses without meal announcement under Scenario 1. Thick lines are median values while shaded areas embrace the $15 \%$ and $85 \%$ percentiles.

\section{G. Glucose target}

The use of different glucose targets during nocturnal and diurnal periods was reported in [14]. This is a safety measure to reduce the risk of nocturnal hypoglycemia. In this work, a glucose target of $G_{r e f}=130 \mathrm{mg} / \mathrm{dL}$ is set for the nocturnal period (between $23 \mathrm{~h}$ and $4 \mathrm{~h}$ ), whereas $G_{r e f}=100 \mathrm{mg} / \mathrm{dL}$ is set during the rest of the day. Notice that the mean values of the noctural and diurnal target zones reported in [14] are similar to the target values chosen here.

\section{H. Tuning}

The controller gains $k_{j}$ (which determine the closed-loop bandwidth) are tuned in relation to the tracking differentiator bandwidth, $\omega$, so that they are the same. A simple way to achieve this is to select the gains as $k_{1}=\omega^{4}, k_{2}=4 \omega^{3}, k_{3}=$ $6 \omega^{2}$ and $k_{4}=4 \omega$. Recall that the CGM readings are available every $5 \mathrm{~min}$, that is, at rate of $1 / 5 \mathrm{~min}^{-1}$, which already imposes a limitation on the achievable bandwidth. Based on this limitation, a closed-loop bandwidth of $\omega=1 / 10 \mathrm{~min}^{-1}$ is selected. Regarding $\gamma_{1}, \gamma_{2}, \gamma_{3}$, those are selected by trial and error to avoid insulin overdose without loosing too much performance. Recall that the factor $\gamma_{1}$ inhibits the control action based on the insulin amount at the first compartment and hence is the fastest inhibition of all three. Therefore, high values of $\gamma_{1}$ have a large impact on performance in the early postprandial period. Keeping that in mind, the value of $\gamma_{1}$ is chosen as small as possible. On the other hand, larger values of $\gamma_{3}$ can be set without affecting performance significantly because its effect takes place after the postprandial peak. The
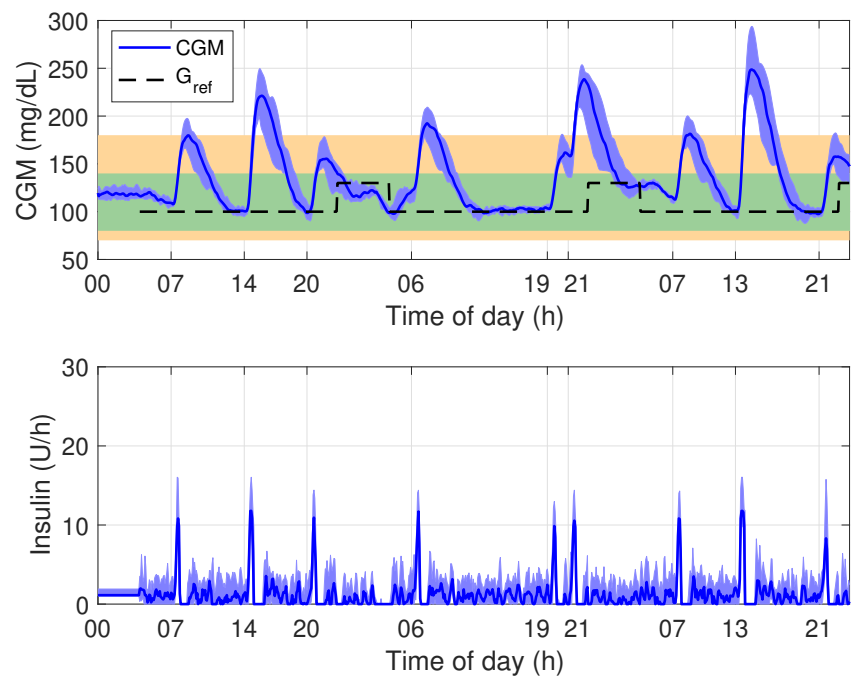

Fig. 2. Closed-loop responses without meal announcement under Scenario 2. Thick lines are median values while shaded areas embrace the $15 \%$ and $85 \%$ percentiles.

tuning is performed through simulations with the entire cohort of patients, in which a meal with a carbohydrate content of $70 \mathrm{~g}$ is given. The population values are finally selected as $\gamma_{1}=0.05, \gamma_{2}=0.2$ and $\gamma_{3}=100$, in order to avoid undershoot in the glucose response (respect to the fasting value). This tuning is conservative as it ensures safe operating conditions for every subject of the cohort.

\section{Optional hybrid mode}

Without meal announcement, the proposed control system estimates disturbances and delivers insulin accordingly, based solely on CGM measurements. However, if desired, an AP system should allow the patient the possibility to announce meals. In that case, the control law (9) is modified by adding up the term $u_{\text {bolus }}(t)=\mathrm{CHO} / \mathrm{CR} \cdot \delta\left(t-t_{\text {meal }}\right)$, where $\mathrm{CHO}$ is the patient's carbohydrate content estimation, $\delta(\cdot)$ is the Dirac delta function and $t_{\text {meal }}$ is the time when the meal is announced. Notice that the bolus is computed using only the meal size and there is no correction term due to neither the deviation between the glucose target and the current one nor insulin-on-board. The injection of an insulin bolus causes a rapid increase in the estimated compartmental and plasma insulin, leading to a fast inhibition. This mechanism plays the same role as the insulin on board limitation reported in other strategies [6], [14].

\section{RESULTS}

In this section, the proposed strategy is validated in silico under three different scenarios. Scenarios 1 and 2 are evaluated 


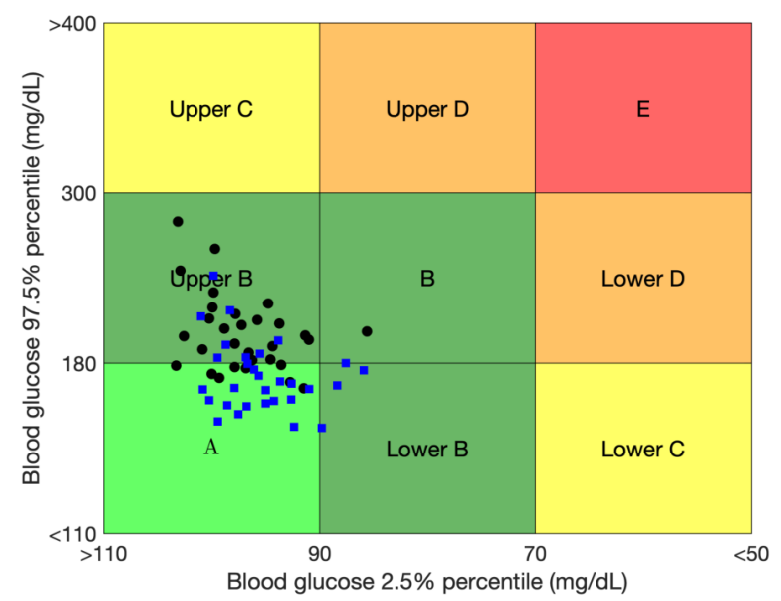

Fig. 3. CVGA plot of comparing the closed-loop responses under Scenario 1 with (blue squares) and without (black circles) meal announcement. Each of the points at the graph is computed upon $24 \mathrm{~h}$ of CGM readings.

using the distribution version of the UVa/Padova simulator [33]. In order to assess robustness to intra-patient variablity, a modified version (described below in Section III.C) of the simulator was used to evaluate Scenario 3. In all three scenarios, closed-loop control with and without meal announcement was considered. In the case of announced meals, CHO misestimation has been simulated according to the study carried out in [47] (see the curve fitted by regression analysis in Fig. 3 therein), which basically reports a tendency to overestimate small meals and underestimate the large ones. In every simulation, open-loop control with basal insulin rate is applied during the first $4 \mathrm{~h}$, after which the controller takes over the insulin delivery [19]. The fasting state of each subject is taken to start the simulation.

\section{A. Scenario 1}

Scenario 1, taken from [19], contains medium-size meals with a $\mathrm{CHO}$ content up to $65 \mathrm{~g}$ (see Table I). Breakfast, lunch and dinner are considered during three consecutive days. The closed-loop responses (median and $15 \%-85 \%$ percentiles) without meal announcement for all in silico subjects of the distribution version of the UVa/Padova simulator under Scenario 1 are shown in Fig. 1. One can see that the proposed controller has good performance but it still avoids drops near the hypoglycemic range. Detailed performance indices of the 3-day period can be found in Table II. The overall performance in this scenario with medium-size meals is very good, scoring a median time-in-range of $90 \%$ and avoiding hypoglycemia. An average time-in-range of $83 \%$ was reported in [19] for the same scenario, although the size of the virtual cohort was substantially larger. Another simulation is performed considering the same scenario with meal announcement. The comparison is illustrated by means of a Control-Variability Grid Analysis (CVGA) plot in Fig. 3. Each of the points depicted in the CVGA plot corresponds to the $2.5 \%$ and $97.5 \%$ distribution of CGM data over a $24 \mathrm{~h}$ period [48]. One can see how the points in the CVGA are shifted downwards when

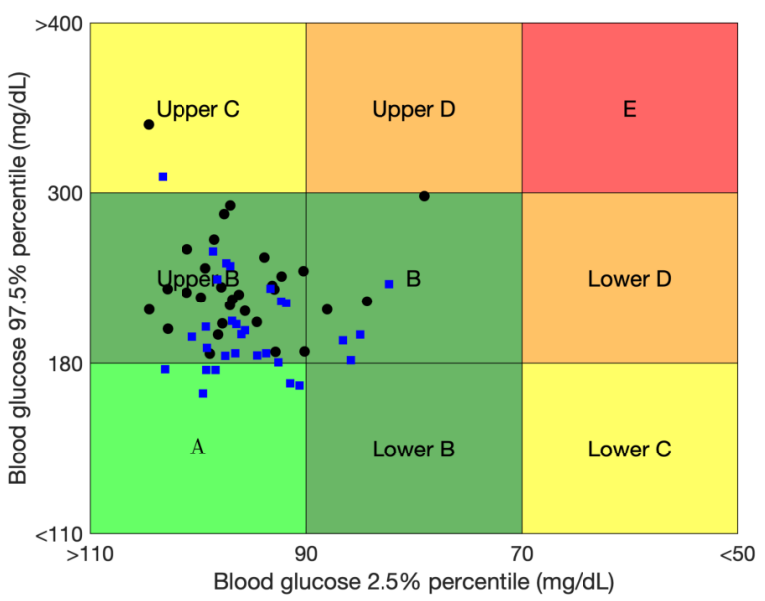

Fig. 4. CVGA plot of comparing the closed-loop responses under Scenario 2 with (blue squares) and without (black circles) meal announcement. Each of the points at the graph is computed upon $24 \mathrm{~h}$ of CGM readings.

meals are announced in spite of the $\mathrm{CHO}$ mismatch, leading also to an outstanding median time-in-range of $99 \%$.

\section{B. Scenario 2}

Scenario 2 is based on the previous one and it includes the following challenging features: i.) large-size meals with CHO content up to $100 \mathrm{~g}$ are included (see Table I); ii.) a fasting period is considered on the second day in which lunch is skipped; iii.) a fairly large snack is given shortly before dinner on the second day ; and iv.) a nocturnal hypoglycemic episode is simulated by introducing an undetected insulin bolus of $2 \mathrm{U}$ the first night at $2 \mathrm{AM}$. The closed-loop responses under Scenario 2 are shown in Fig. 2. At first glance, the overall perfornance is also good in spite of the simulated nocturnal hypoglycemic episode. The postprandial excursions get further into the hypoglycemic range due to the large-size
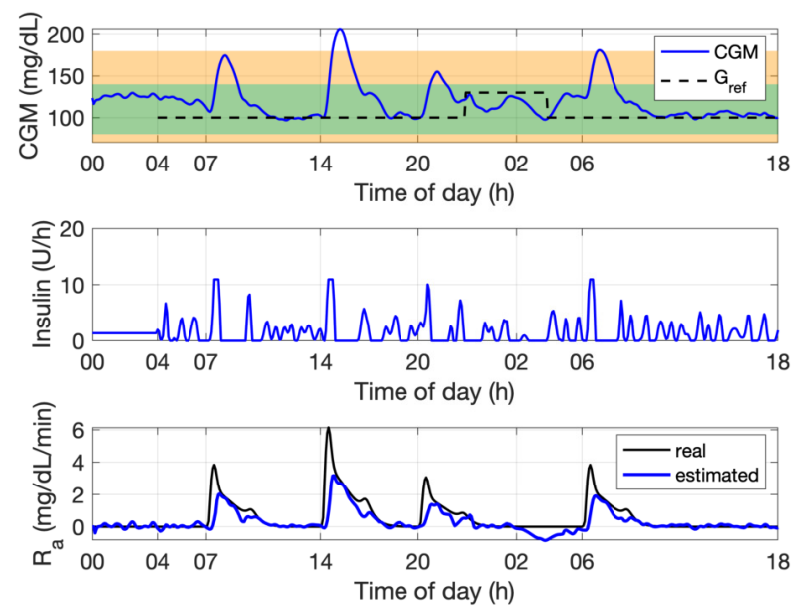

Fig. 5. Detail of the first $42 \mathrm{~h}$ of the closed-loop response under Scenario 2 (3-day simulation with large-size meals and nocturnal hypoglycemia), for the virtual subject \#2 of the distribution version of the UVa/Padova simulator. 
TABLE II

OVERALL PERFORMANCE INDICES (MEDIAN VALUES WITH 15\% AND 85\% PERCENTILES) FOR THE IN SILICO EVALUATION

\begin{tabular}{lllllll}
\hline & \multicolumn{2}{c}{ Scenario 1 } & \multicolumn{2}{c}{ Scenario 2 } & \multicolumn{2}{c}{ Scenario 3 } \\
& Announced & Unannouced & Announced & Unannouced & Announced & Unannouced \\
\hline Mean BG & $128(123,130)$ & $136(125,140)$ & $130(123,132)$ & $138(126,142)$ & $135(128,144)$ & $144(131,153)$ \\
$\% \in[70,180]$ & $99(97,100)$ & $90(83,98)$ & $93(90,98)$ & $85(83,95)$ & $88(80,94)$ & $80(74,88)$ \\
$\% \in[80,140]$ & $71(69,84)$ & $65(59,76)$ & $72(71,79)$ & $65(59,74)$ & $66(58,71)$ & $57(50,68)$ \\
$\%<70$ & $0.0(0.0,0.0)$ & $0.0(0.0,0.0)$ & $0.0(0.0,0.0)$ & $0.0(0.0,0.0)$ & $0.0(0.0,0.1)$ & $0.0(0.0,0.0)$ \\
$\%<60$ & $0.0(0.0,0.0)$ & $0.0(0.0,0.0)$ & $0.0(0.0,0.0)$ & $0.0(0.0,0.0)$ & $0.0(0.0,0.0)$ & $0.0(0.0,0.0)$ \\
$\%<50$ & $0.0(0.0,0.0)$ & $0.0(0.0,0.0)$ & $0.0(0.0,0.0)$ & $0.0(0.0,0.0)$ & $0.0(0.0,0.0)$ & $0.0(0.0,0.0)$ \\
$\%>250$ & $0.0(0.0,0.0)$ & $0.0(0.0,0.0)$ & $0.0(0.0,0.5)$ & $0.4(0.0,1.7)$ & $0.2(0.0,2.0)$ & $1.3(0.1,4.2)$ \\
$\%>300$ & $0.0(0.0,0.0)$ & $0.0(0.0,0.0)$ & $0.0(0.0,0.0)$ & $0.0(0.0,0.0)$ & $0.0(0.0,0.1)$ & $0.0(0.0,0.6)$ \\
HBGI & $1.3(0.8,1.6)$ & $2.5(1.2,2.8)$ & $1.9(1.1,2.1)$ & $3.2(1.5,3.8)$ & $2.6(1.8,4.0)$ & $3.8(2.4,5.0)$ \\
LBGI & $0.1(0.0,0.2)$ & $0.1(0.0,0.2)$ & $0.1(0.1,0.2)$ & $0.1(0.1,0.2)$ & $0.2(0.1,0.2)$ & $0.1(0.0,0.2)$ \\
\hline
\end{tabular}

meals. However, the performance indices (see Table II) are still fairly good, scoring a median time-in-range of $86 \%$ and avoiding hypoglycemia. The comparison with the announced scenario is again illustrated by means of a CVGA plot in Fig. 4, where improvement is also observed, achieving $93 \%$ of median time-in-range.

In order to provide a better insight in how the observer works, a detailed view of the individual closed-loop response of patient \#2 for the first $42 \mathrm{~h}$ is shown in Fig. 5. The meal glucose rate of appearance (black) and its estimation (blue) are depicted at the bottom plot. The estimated value aggregates any unexpected change in the glucose level. That includes the effect of meals but also model uncertainties and other disturbances such as induced nocturnal hypoglycemia. One can see how the estimation drops below zero shortly after $2 \mathrm{AM}$ (when the $2 \mathrm{U}$ insulin bolus is delivered) leading to a pump suspension that mitigates that glucose drop. On the other hand, one can also appreciate in this plot the delay between the real meal glucose rate and its estimation, which is due to the sensing delay and observer dynamics. At this point, it should be remarked that this observer would also estimate a glucose drop due to exercise, which could be compensated using specific strategies proposed in the literature [49].

\section{Scenario 3}

Finally, a third scenario is considered to evaluate the robustness of the proposed strategy. Scenario 3 has a duration of 30 days, with three randomized meals per day of $65 \mathrm{~g}$, $70 \mathrm{~g}, 65 \mathrm{~g}$ with a $\mathrm{CV}=10 \%$ delivered at $7 \mathrm{~h}, 14 \mathrm{~h}$ and $21 \mathrm{~h}$ with STD $=60 \mathrm{~min}$, respectively. The scenario also takes into account large intra-subject variability by varying insulin absorption model parameters up to $\pm 30 \%$ from one meal intake to another, and insulin sensitivity parameters up to $\pm 30 \%$ with a sinusoidal pattern along the day [50]. For intrasubject meal variability, the ten sets of meal model parameters of the cohort were randomly assigned to each other at each meal intake. This is a very challenging scenario in which 35 out of 90 meals have a $\mathrm{CHO}$ content over $70 \mathrm{~g}$, being the average $66.5 \mathrm{~g}$ per meal. The results of this simulation are displayed by means of a CVGA plot in Fig. 6. Without meal announcement, a few number of samples lay on the out of the $\mathrm{A}+\mathrm{B}$ zones, of which approximately $77 \%$ correspond to patient \#7 (as pointed out in [38], this patient has an insulin sensitivity that is not coherent with its TDI). Nevertheless, the

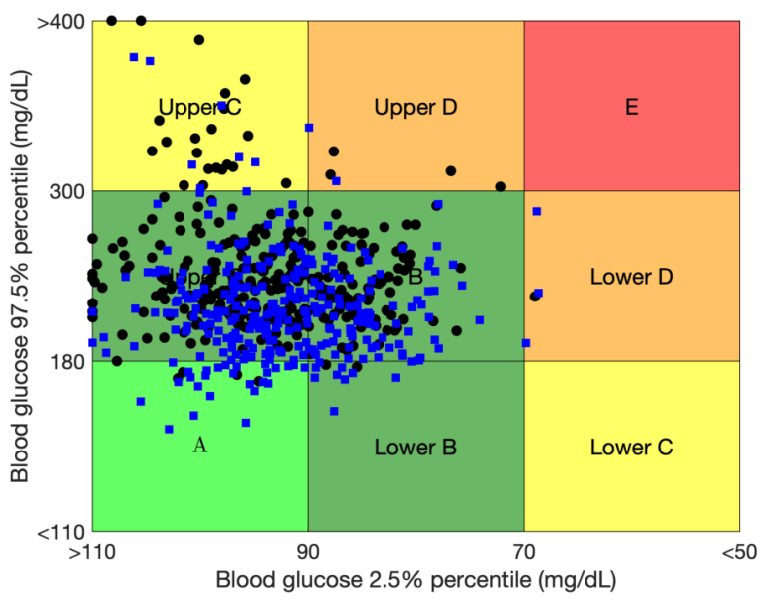

Fig. 6. CVGA plot of comparing the closed-loop responses under Scenario 3 with (blue squares) and without (black circles) meal announcement. Each of the points at the graph is computed upon $24 \mathrm{~h}$ of CGM readings.

overall counting indicates that $91 \%$ of the samples lay within the $\mathrm{A}+\mathrm{B}$ zones in the unannounced case, which is not far from the $95 \%$ scored in the announced case. It should be pointed out that the tendency to hyperglycemia in spite of meal boluses is due to the fact that the simulated uncertainty in $\mathrm{CHO}$ counting severely under-estimates large meals. As indicated in Table II, the unannounced strategy leads to a median time-in-range of $80 \%$, which is increased up to $88 \%$ when meals are announced.

\section{CONCLusions}

A novel control algorithm for artificial pancreas system has been proposed in this work, based on a disturbance observer and feedforward compensation. It has been shown how the disturbance observer is capable of estimating disturbances that cause unexpected variations in glucose levels. That includes the effect of meals but also model uncertainties or sudden drops that could result in hypoglycemia. The tuning of the proposed controller is feasible as it is based on a priori information of each subject. For unannounced meals, the results are promising, showing a median time-in-range of $80 \%$ in a challenging 30-day scenario with high carbohydrate content and large intra-subject variability. Results show this performance is further improved by $+10 \%$ in the same scenario when meals are announced in spite of severe $\mathrm{CHO}$ misestimation. 
While successful postprandial control with meal announcement has been reported, exercise was not discussed in this work, which is a another major challenge towards a fully automated artificial pancreas. Future research will be directed to exploit the information provided by the disturbance observer in order to mitigate hypoglycemia induced by exercise.

\section{REFERENCES}

[1] American Diabetes Association, "Diagnosis and classification of diabetes mellitus," Diabetes Care, vol. 37, Supplement 1, pp. S81-S90, 2014.

[2] E.-F. Pfeiffer, C. Thum, and A. Clemens, "The artificial beta cell-a continuous control of blood sugar by external regulation of insulin infusion (glucose controlled insulin infusion system)," Hormone and Metabolic Research, vol. 6, no. 05, pp. 339-342, 1974.

[3] A. Albisser, B. Leibel, T. Ewart, Z. Davidovac, C. Botz, and W. Zingg, "An artificial endocrine pancreas," Diabetes, vol. 23, no. 5, pp. 389-396, 1974.

[4] E. J. Fogt, L. M. Dodd, E. Jenning, and A. H. Clemens, "Development and evaluation of a glucose analyzer for a glucose controlled insulin infusion system (biostator)." Clinical Chemistry, vol. 24, no. 8, pp. 1366-1372, 1978

[5] R. Hovorka, "Continuous glucose monitoring and closed-loop systems," Diabetic Medicine, vol. 23, no. 1, pp. 1-12, 2006

[6] G. M. Steil, C. C. Palerm, N. Kurtz, G. Voskanyan, A. Roy, S. Paz, and F. R. Kandeel, "The effect of insulin feedback on closed loop glucose control," The Journal of Clinical Endocrinology \& Metabolism, vol. 96, no. 5, pp. 1402-1408, 2011.

[7] H. Thabit, A. Lubina-Solomon, M. Stadler, L. Leelarathna, E. Walkinshaw, A. Pernet, J. M. Allen, A. Iqbal, P. Choudhary, K. Kumareswaran et al., "Home use of closed-loop insulin delivery for overnight glucose control in adults with type 1 diabetes: a 4-week, multicentre, randomised crossover study," The Lancet Diabetes \& Endocrinology, vol. 2, no. 9, pp. 701-709, 2014.

[8] B. Kovatchev, P. Cheng, S. M. Anderson, J. E. Pinsker, F. Boscari, B. A. Buckingham, F. J. Doyle III, K. K. Hood, S. A. Brown, M. D. Breton et al., "Feasibility of long-term closed-loop control: a multicenter 6month trial of 24/7 automated insulin delivery," Diabetes Technology \& Therapeutics, vol. 19, no. 1, pp. 18-24, 2017.

[9] S. K. Garg, S. A. Weinzimer, W. V. Tamborlane, B. A. Buckingham, B. W. Bode, T. S. Bailey, R. L. Brazg, J. Ilany, R. H. Slover, S. M Anderson et al., "Glucose outcomes with the in-home use of a hybrid closed-loop insulin delivery system in adolescents and adults with type 1 diabetes," Diabetes Technology \& Therapeutics, vol. 19, no. 3, pp. 155-163, 2017.

[10] A. Weisman, J.-W. Bai, M. Cardinez, C. K. Kramer, and B. A. Perkins, "Effect of artificial pancreas systems on glycaemic control in patients with type 1 diabetes: a systematic review and meta-analysis of outpatient randomised controlled trials," The lancet Diabetes \& Endocrinology, vol. 5, no. 7, pp. 501-512, 2017.

[11] R. S. Parker, F. J. Doyle, and N. A. Peppas, "A model-based algorithm for blood glucose control in type 1 diabetic patients," IEEE Transactions on Biomedical Engineering, vol. 46, no. 2, pp. 148-157, 1999.

[12] R. Hovorka, V. Canonico, L. J. Chassin, U. Haueter, M. Massi-Benedetti, M. O. Federici, T. R. Pieber, H. C. Schaller, L. Schaupp, T. Vering et al., "Nonlinear model predictive control of glucose concentration in subjects with type 1 diabetes," Physiological Measurement, vol. 25, no. 4, p. 905, 2004.

[13] K. Turksoy, E. S. Bayrak, L. Quinn, E. Littlejohn, and A. Cinar, "Multivariable adaptive closed-loop control of an artificial pancreas without meal and activity announcement," Diabetes Technology \& Therapeutics, vol. 15, no. 5, pp. 386-400, 2013.

[14] R. Gondhalekar, E. Dassau, and F. J. Doyle III, "Periodic zone-MPC with asymmetric costs for outpatient-ready safety of an artificial pancreas to treat type 1 diabetes," Automatica, vol. 71, pp. 237-246, 2016.

[15] G. M. Steil, K. Rebrin, C. Darwin, F. Hariri, and M. F. Saad, "Feasibility of automating insulin delivery for the treatment of type 1 diabetes," Diabetes, vol. 55, no. 12, pp. 3344-3350, 2006.

[16] G. Marchetti, M. Barolo, L. Jovanovič, H. Zisser, and D. E. Seborg, "A feedforward-feedback glucose control strategy for type 1 diabetes mellitus," Journal of Process Control, vol. 18, no. 2, pp. 149-162, 2008.
[17] R. Mauseth, I. B. Hirsch, J. Bollyky, R. Kircher, D. Matheson, S. Sanda, and C. Greenbaum, "Use of a fuzzy logic controller in a closed-loop artificial pancreas," Diabetes Technology \& Therapeutics, vol. 15, no. 8, pp. 628-633, 2013.

[18] R. Nimri, I. Muller, E. Atlas, S. Miller, A. Fogel, N. Bratina, O. Kordonouri, T. Battelino, T. Danne, and M. Phillip, "Md-logic overnight control for 6 weeks of home use in patients with type 1 diabetes: randomized crossover trial," Diabetes Care, vol. 37, no. 11, pp. 30253032, 2014.

[19] P. H. Colmegna, R. S. Sánchez-Peña, R. Gondhalekar, E. Dassau, and F. J. Doyle, "Switched LPV glucose control in type 1 diabetes," IEEE Transactions on Biomedical Engineering, vol. 63, no. 6, pp. 1192-1200, 2016.

[20] R. Sánchez-Peña, P. Colmegna, L. Grosembacher, M. Breton, H. De Battista, F. Garelli, W. H. Belloso, E. Campos-Nánez, V. Simonovich, V. Beruto et al., "Artificial pancreas: first clinical trials in argentina," IFAC-PapersOnLine, vol. 50, no. 1, pp. 7731-7736, 2017.

[21] J. Xie and Q. Wang, "A variable state dimension approach to meal detection and meal size estimation: in silico evaluation through basalbolus insulin therapy for type 1 diabetes," IEEE Transactions on Biomedical Engineering, vol. 64, no. 6, pp. 1249-1260, 2016.

[22] S. Samadi, K. Turksoy, I. Hajizadeh, J. Feng, M. Sevil, and A. Cinar, "Meal detection and carbohydrate estimation using continuous glucose sensor data," IEEE journal of Biomedical and Health Informatics, vol. 21, no. 3, pp. 619-627, 2017.

[23] I. Hajizadeh, M. Rashid, K. Turksoy, S. Samadi, J. Feng, M. Sevil, N. Hobbs, C. Lazaro, Z. Maloney, E. Littlejohn et al., "Incorporating unannounced meals and exercise in adaptive learning of personalized models for multivariable artificial pancreas systems," Journal of Diabetes Science and Technology, vol. 12, no. 5, pp. 953-966, 2018.

[24] S. Samadi, M. Rashid, K. Turksoy, J. Feng, I. Hajizadeh, N. Hobbs, C. Lazaro, M. Sevil, E. Littlejohn, and A. Cinar, "Automatic detection and estimation of unannounced meals for multivariable artificial pancreas system," Diabetes Technology \& Therapeutics, vol. 20, no. 3, pp. 235-246, 2018

[25] C. Ramkissoon, P. Herrero, J. Bondia, and J. Vehi, "Unannounced meals in the artificial pancreas: detection using continuous glucose monitoring," Sensors, vol. 18, no. 3, p. 884, 2018.

[26] R. Gondhalekar, E. Dassau, and F. J. Doyle III, "Velocity-weighting \& velocity-penalty mpc of an artificial pancreas: Improved safety \& performance," Automatica, vol. 91, pp. 105-117, 2018.

[27] J.-L. Guzmán and T. Hägglund, "Simple tuning rules for feedforward compensators," Journal of Process Control, vol. 21, no. 1, pp. 92-102, 2011.

[28] W.-H. Chen, J. Yang, L. Guo, and S. Li, "Disturbance-observer-based control and related methods: an overview," IEEE Transactions on Industrial Electronics, vol. 63, no. 2, pp. 1083-1095, 2016.

[29] R. Sanz, P. Garcia, Q.-C. Zhong, and P. Albertos, "Robust control of quadrotors based on an uncertainty and disturbance estimator," Journal of Dynamic Systems, Measurement and Control, vol. 138, no. 7, p. 071006, 2016.

[30] I. Sala, J.-L. Díez, and J. Bondia, "Generalized extended state observer design for the estimation of the rate of glucose appearance in artificial pancreas," in 2018 European Control Conference (ECC). IEEE, 2018, pp. 2393-2398.

[31] I. Sala, J.-L. Díez, B. Ricarte, and J. Bondia, "Sliding-mode disturbance observers for an artificial pancreas without meal announcement," Journal of Process Control, vol. 78, pp. 68-77, 2019.

[32] S. S. Kanderian, S. A. Weinzimer, and G. M. Steil, "The identifiable virtual patient model: comparison of simulation and clinical closed-loop study results," Journal of Diabetes Science and Technology, vol. 6, no. 2, pp. 371-379, 2012.

[33] C. Dalla Man, F. Micheletto, D. Lv, M. Breton, B. Kovatchev, and C. Cobelli, "The UVA/PADOVA type 1 diabetes simulator: new features," Journal of Diabetes Science and Technology, vol. 8, no. 1, pp. 26-34, 2014.

[34] J. Walsh, R. Roberts, and T. Bailey, "Guidelines for insulin dosing in continuous subcutaneous insulin infusion using new formulas from a retrospective study of individuals with optimal glucose levels," Journal of Diabetes Science and Technology, vol. 4, no. 5, pp. 1174-1181, 2010.

[35] M. Messori, G. P. Incremona, C. Cobelli, and L. Magni, "Individualized model predictive control for the artificial pancreas: In silico evaluation of closed-loop glucose control," IEEE Control Systems Magazine, vol. 38, no. 1, pp. 86-104, 2018.

[36] M. Di Ferdinando, P. Pepe, P. Palumbo, S. Panunzi, and A. De Gaetano, "Semiglobal sampled-data dynamic output feedback controller for the 
glucose-insulin system," IEEE Transactions on Control Systems Technology, 2018.

[37] K. van Heusden, E. Dassau, H. C. Zisser, D. E. Seborg, and F. J. Doyle III, "Control-relevant models for glucose control using a priori patient characteristics," IEEE Transactions on Biomedical Engineering, vol. 59, no. 7, pp. 1839-1849, 2011.

[38] P. Colmegna, R. S. Sánchez-Peña, and R. Gondhalekar, "Linear parameter-varying model to design control laws for an artificial pancreas," Biomedical Signal Processing and Control, vol. 40, pp. 204-213, 2018.

[39] H. Lin and P. J. Antsaklis, "Stability and stabilizability of switched linear systems: a survey of recent results," IEEE Transactions on Automatic Control, vol. 54, no. 2, pp. 308-322, 2009.

[40] G. C. Goodwin, S. F. Graebe, M. E. Salgado et al., Control system design. Prentice Hall New Jersey, 2001, vol. 240.

[41] W. P. Dayawansa and C. F. Martin, "A converse lyapunov theorem for a class of dynamical systems which undergo switching," IEEE Transactions on Automatic Control, vol. 44, no. 4, pp. 751-760, 1999.

[42] H. K. Khalil, "Nonlinear systems," Upper Saddle River, 2002.

[43] B.-Z. Guo and Z.-L. Zhao, "On convergence of tracking differentiator," International Journal of Control, vol. 84, no. 4, pp. 693-701, 2011.

[44] J. Bondia, S. Romero-Vivo, B. Ricarte, and J. L. Diez, "Insulin estimation and prediction: a review of the estimation and prediction of subcutaneous insulin pharmacokinetics in closed-loop glucose control," IEEE Control Systems Magazine, vol. 38, no. 1, pp. 47-66, 2018.

[45] C. C. Palerm, "Physiologic insulin delivery with insulin feedback: a control systems perspective," Computer Methods and Programs in Biomedicine, vol. 102, no. 2, pp. 130-137, 2011.

[46] J. L. Ruiz, J. L. Sherr, E. Cengiz, L. Carria, A. Roy, G. Voskanyan, W. V. Tamborlane, and S. A. Weinzimer, "Effect of insulin feedback on closed-loop glucose control: a crossover study," Journal of Diabetes Science and Technology, vol. 6, no. 5, pp. 1123-1130, 2012.

[47] T. Kawamura, C. Takamura, M. Hirose, T. Hashimoto, T. Higashide, Y. Kashihara, K. Hashimura, and H. Shintaku, "The factors affecting on estimation of carbohydrate content of meals in carbohydrate counting," Clinical Pediatric Endocrinology, vol. 24, no. 4, pp. 153-165, 2015.

[48] L. Magni, D. M. Raimondo, C. D. Man, M. Breton, S. Patek, G. De Nicolao, C. Cobelli, and B. P. Kovatchev, "Evaluating the efficacy of closed-loop glucose regulation via control-variability grid analysis," Journal of Diabetes Science and Technology, vol. 2, no. 4, pp. 630-635, 2008.

[49] A. Beneyto, A. Bertachi, J. Bondia, and J. Vehi, "A new blood glucose control scheme for unannounced exercise in type 1 diabetic subjects," IEEE Transactions on Control Systems Technology, 2018.

[50] N. Resalat, J. El Youssef, N. Tyler, J. Castle, and P. G. Jacobs, "A statistical virtual patient population for the glucoregulatory system in type 1 diabetes with integrated exercise model," PLoS One, vol. 14, no. 7, p. e0217301, 2019

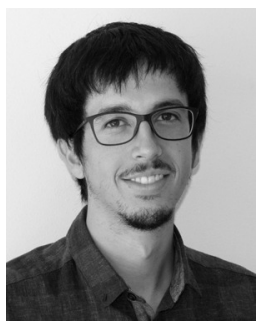

Ricardo Sanz received his M.Sc. degree in Aerospace from the Universitat Politècnica de Valtència (UPV), Spain, in 2013. He has been exchange student at the Royal Institute of Technology, Stockholm, Sweden and at the Georgia Institute of Technology, Atlanta, GA, USA. He received his M.Sc. degree in Robotics and Automation in 2014 and his Ph.D. in Automatic Control in 2018, both from the School of Industrial Engineering at the UPV. He has been a visiting researcher at the Université de Technologie de Compiègne, France, the University of Zhejiang, Hangzhou, China and the University of California San Diego, CA, USA. His current research interests include control of time-delay systems, predictive control, disturbance observers and biomedical applications.

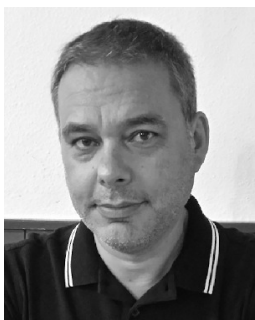

Pedro García received the Ph.D. degree in Control Systems and Industrial Computing from the Universitat Politècnica de València, València, Spain, in 2007, where he is currently an Associate Professor within the Department of Systems Engineering and Control. He has been a visiting researcher at the Lund Institute of Technology, Sweden, the Université de Technologie de Compiègne, France, the University of Florianopolis, Brazil, the University of Sheffield, UK, and the University of Zhejiang, Hangzhou, China. He has coauthored one book, and more than 80 refereed journal and conference papers. His current research interests include control of time-delay systems, disturbance observers and control in type 1 diabetes.

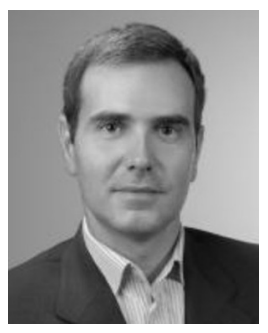

José Luis Díez received the M.Sc degree in Industrial Engineering in 1995 and the Ph.D. degree in Control Engineering in 2003, both from the Universitat Politècnica de València (UPV), València, Spain. $\mathrm{He}$ is currently an Associate Professor and he has been lecturing since 1995 with the Department of Systems Engineering and Control of the UPV. He has been a visiting researcher at the Politecnico di Milano, Italy, and the Illinois Institute of Technology, Chicago, IL, USA. He is Deputy Director of the Iberoamerican Journal of Control and Industrial Informatics (RIAI) and he has coauthored more than 100 refereed journal and conference papers. His research interests include complex systems modeling and identification, data mining and intelligent control in type 1 diabetes.

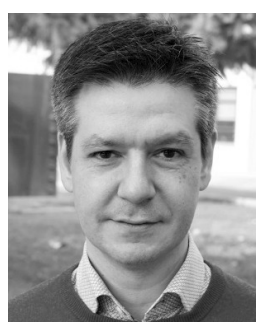

Jorge Bondia received the Ph.D. degree in Computer Science from the Universitat Politècnica de València (UPV), València, Spain, in 2002. In 1995, he started lecturing with the Department of Systems Engineering and Control at the UPV, where he is Full Professor since 2017. He is Principal Investigator at the Diabetes and Associated Metabolic Diseases Networking Biomedical Research Centre (CIBERDEM), Madrid, Spain, since 2018, and coordinator of the Spanish Consortium on Artificial Pancreas and Diabetes Technology. He has authored more than 60 journal articles and more than 120 conference contributions in the area of diabetes technology. His research interests include modeling and control in type 1 diabetes, biomedical applications, systems medicine, uncertain dynamical systems, monotone systems and robust control. 